\title{
Structural and Spectral (IR, NMR and UV/Visible) Properties of Newly Designed Boronic Acid Derivatives Containing D03A Sensitive to Uranyl Ion: A DFT and TD-DFT Study
}

\author{
Kübra Sarikavak, Fatma Sevin* \\ Chemistry Department, Faculty of Science, Hacettepe University, Ankara, Turkey \\ Email: *sevin@hacettepe.edu.tr
}

How to cite this paper: Sarikavak, K. and Sevin, F. (2017) Structural and Spectral (IR, NMR and UV/Visible) Properties of Newly Designed Boronic Acid Derivatives Containing DO3A Sensitive to Uranyl Ion: A DFT and TD-DFT Study. Computational Chemistry, 5, 145-158.

https://doi.org/10.4236/cc.2017.54012

Received: September 10, 2017

Accepted: October 13, 2017

Published: October 16, 2017

Copyright $\odot 2017$ by authors and Scientific Research Publishing Inc. This work is licensed under the Creative Commons Attribution International License (CC BY 4.0).

http://creativecommons.org/licenses/by/4.0/

\begin{abstract}
In this study, the structural, electronic and optical properties of DO3A-based boronic acid derivates with and without $\mathrm{UO}_{2}^{2+}$ ion are studied by density functional calculations with M062X/6 - $31 \mathrm{~g}+(\mathrm{d})$ method, in aqueous media. The quantum chemical properties such as $\mathrm{E}_{\text {номо }}, \mathrm{E}_{\mathrm{LUMO}}$, the energy gap $(\Delta \mathrm{E})$, chemical potential $(\mu)$, hardness $(\eta)$ are also performed. The theoretical essen-

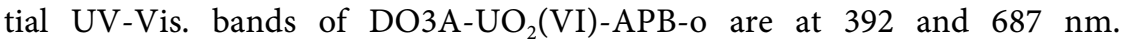
DO3A-UO ${ }_{2}$ (VI)-ABB-o structure has two main bands at 393 and $650 \mathrm{~nm}$. In general, the bathochromic shift occurs and the HOMO-LUMO energy gap decreases to about $2 \mathrm{eV}$, by binding $\mathrm{UO}_{2}^{2+}$ ion in three different media. The notable shifts in NMR spectrums have been found on $\alpha$-carbon of carbonyl group, ring carbons and amide protons. In IR-spectrums, the prominent peaks belong to $\mathrm{BO}-\mathrm{H}$ and $\mathrm{N}-\mathrm{H}$ of amide stretching vibrations of calculated structures.
\end{abstract}

\section{Keywords}

Uranium, DO3A, Benzimidazole, DFT

\section{Introduction}

Boronic acid derivatives have attracted attention from many applications due to their unique properties and are widely used in Diels Alder reactions [1], asymmetric synthesis [2], cross-coupling reactions [3], activation of carboxylic acids 
and pattern in synthesis [4]. Due to the binding properties of boronic acid derivatives, they have been used in sensor studies [5]. Since using 2-arylboronic acid as a chemosensor for polyols, it has been used as a fluorescence probe in many studies [6]. Some organoboron compounds have been developed for fluoride ion sensors [7]. Carotenoid derivatives containing $\mathrm{B}(\mathrm{OH})_{2}$ groups have been used as potential sugar sensor [8].

On the other hand, DO3A (tetraazacyclododecane-1,4,7,-three acetic acid) molecules in the scope of this study is a compound known as a cyclin size macro and they can be seen as a chelating agent with higher affinity because of the carbonyl groups [9]. Since these molecules have been synthesized, they often used in several ions complexes as $\mathrm{Ni}^{2+}, \mathrm{Cu}^{2+}, \mathrm{Ca}^{2+}$ and $\mathrm{Gd}^{3+}$ and also particularly yttrium complexes are investigated in a variety of research and applications like therapeutic radiopharmaceuticals [10], positron emission tomography [11], magnetic resonance imaging and medical diagnosis and treatment as high thermodynamic stability features [12].

One of the main applications of these molecules is the formation of the lanthanide complexes clingy by DOTA (tetraazacyclododecane-1,4,7,10-tetra acetic acid) lanthanides bound to the amino and carboxyl groups [13]. DOTA and their complexes have been subjected to various experimental work and theoretical studies in the literature. The study was conducted by Iglesias and different isomers of DOTA and related lanthanide complexes have been investigated in the solvent medium and stable geometries and energies specified with the DFT method [14]. In another study, structural properties of Ln (III) complexes with a tetrapyridine pendant-armed macrocyclic ligand have been investigated by DFT method [15].

As can be seen from the above some mentioned studies, quick and versatile quantitative determination of molecular diagnostics of the mutated gene associated with human disease plays an important role in modern clinical treatment and genomic studies. On the other hand, radioactive waste, such as the uranyl ions consisting of the fuel nuclear power plant and ore beneficiation are carried to the living systems on through land, water and food chains. To solve the uranyl chemistry in living systems, specifically, understanding of interactions with biomolecules can be achieved by calculation studies at the molecular level.

In this sense, new designed DO3A-based sensors containing benzimidazole and phenyl group with boronic acid have been shown in Figure 1. Their stability of uranyl ion and possible structures with DO3A have been calculated in water media and also chemical properties such as $\mathrm{E}_{\text {Hомо }}, \mathrm{E}_{\mathrm{LUMO}}$, the energy gap $(\Delta \mathrm{E})$, chemical potential $(\mu)$, hardness $(\eta)$, softness $(S)$, the absolute electronegativity $(\chi)$ and the electrophilicity index $(\omega)$ have been predicted. Moreover, detections of these complexes in water phase spectroscopic properties (UV-Vis, IR and NMR) have been given.

\section{Method}

All calculations were performed with using the Gaussian [16] 09W and Gauss 


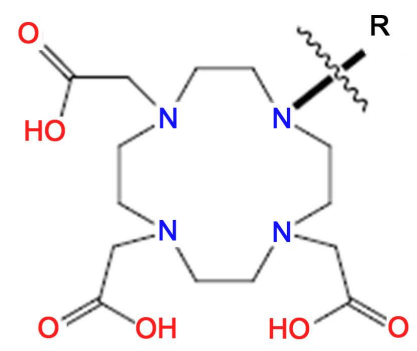

DO3A

$\mathrm{R}$ :

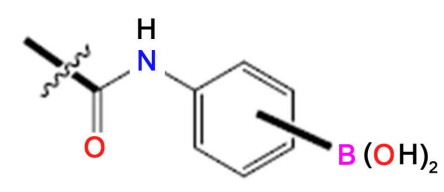

APB-o, $m, p$
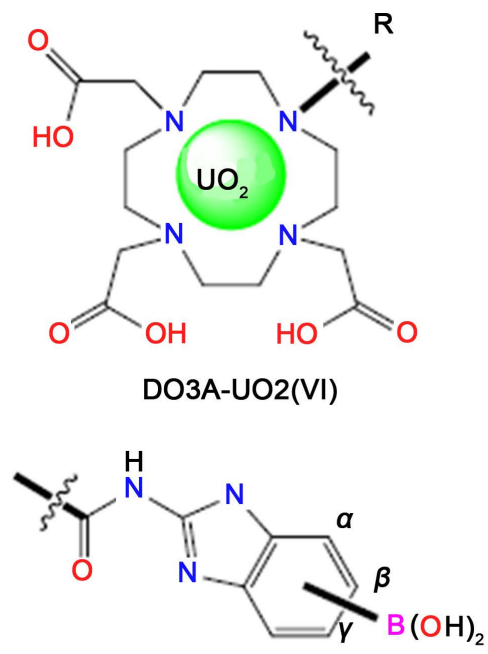

ABB- $\alpha, \beta, \gamma$

Figure 1. Designed DO3A-based sensors containing benzimidazole and phenylgroup.

View 5.0.8 molecular modeling software [17]. Also, ChemCraft program [18] was used to determine the diameter of the examined structures. M062X [19], B3LYP [20] and B3PW91 [21] methods that can be suitable for calculations are selected and compared with experimental absorption value of reference uranium complex [22]. First of all, U (IV) DO3A complex was optimized with selected three methods in the gas phase. Optimization in the solvent phase (methanol) was then carried out and the UV-Vis spectrum was obtained. The calculation results compared with experimental value and show that (Table S1 given in Supporting information) M062X functional was found better than the other selected methods. After determination of the appropriate method, all subsequent calculations performed with M062X functional and $6-31+\mathrm{G}$ (d) [23] basis set. The SARC ZORA basis set was used for the uranium atom [24]. The SMD Solvation Model Density [25] method was chosen to reflect the effect of the water phase on the calculations. The dielectric constant was chosen as the standard value for water $\varepsilon=78.39$ ). Calculations corresponding to acidic medium implemented with nitrogen protonation. The basic medium is provided by the removal of hydrogen atoms in the carboxylic groups on DO3A.

\section{Results and Discussion}

\subsection{D03A-Based Aminophenol and Aminobenzo Imidazolyl Boronic Acid Derivatives with and without $\mathrm{UO}_{2}^{2+}$}

\subsubsection{Stabilities}

After selecting the appropriate method for the calculations, boronic acid-containing derivatives of ortho, para and meta positions of DO3A based sensors have been investigated. Table 1 shows that energy values of DO3AAPB and DO3A-ABB derivatives in gas and water phases.

The calculated energy results show that the o-boronic acid structure, DO3A-APB-o 
Table 1. Total energies of DO3A-APB and DO3A-ABB structures (kcal/mol).

\begin{tabular}{ccccc}
\hline Compound & Gaz phase & Stability & Water phase & Stability \\
\hline DO3A-APB-o & $-1,150,397.88$ & - & $-1,150,446.42$ & - \\
DO3A-APB-m & $-1,150,394.81$ & 3.07 & $-1,150,444.17$ & 2.25 \\
DO3A-APB-p & $-1,150,395.47$ & 2.41 & $-1,150,444.03$ & 2.39 \\
DO3A-ABB- $\alpha$ & $-1,242,992.44$ & - & $-1,243,051.98$ & - \\
DO3A-ABB- $\beta$ & $-1,242,990.76$ & 1.68 & $-1,243,049.84$ & 2.14 \\
DO3A-ABB- $\gamma$ & $-1,242,989.14$ & 3.30 & $-1,243,050.51$ & 1.47 \\
\hline
\end{tabular}

is the more stable than the other positions in the gas phase, about of $2-3$ $\mathrm{kcal} / \mathrm{mol}$ energy values. In the water phase, the same trend was found about 2 $\mathrm{kcal} / \mathrm{mol}$. When the energy of structures in water and gas phases is compared, it has been found that the presence of water in the environment brings about the solvation stability.

Table 1 shows that DO3A-ABB- $\alpha$ structure is more stable than the other positions in the gas and water phases, about of $1.5-3 \mathrm{kcal} / \mathrm{mol}$ energy values.

As a result, DO3A-APB-o and DO3A-ABB- $\alpha$ have been found the most stable within calculated structures and so by choosing these compounds, further calculations have been continued. Optimized DO3A-APB-o and DO3A-ABB- $\alpha$ structures have been presented in Table S2 and Table S3.

\subsubsection{Structural Properties and Molecular Orbitals}

Ortho structures with and without uranyl ion have been examined in terms of their structural, electronic and spectroscopic properties in acidic, basic and neutral media. While the acidic medium was formed by protonation of the nitrogen (N6), the basic medium was formed by the removal of the carboxylic proton located on DO3A. The numbered structures of some selected atoms of all calculated of DO3A-based sensors with/without uranyl ion are presented, acidic, neutral and basic media, in Figure 2 and Table 2.

C-C bond lengths of the target compounds are in the range of $1.530-1.534 \AA$. This bond length with uranyl ion is changed from 1.564 to $1.576 \AA$. For compound without uranyl ion, the bond length between nitrogen and carbon in that fragment vary from 1.455 to $1.476 \AA$. For a compound with uranyl ion it increases to $1.464-1.528 \AA$.

The bond length between uranyl and nitrogen are between 2.246 and 1.2684 $\AA$ A. For uranyl-free structures, the bond length of boron and hydroxide is between 1.363 and $1.371 \AA$, while bond length with uranium slightly increased to range of 1.377 and $1.399 \AA$, within indicates three media. Diameters of related structures in the water medium have been determined theoretically and given in Table S4. DO3A-APB-oand DO3A-ABB- $\alpha$ structures have been found to be about $11 \AA$ in three mediums. The diameter of the structures with uranyl ion is wider than the others, $1 \AA$.

When the charges of the DO3A-APB-o structures in Table 3 are examined, 
Table 2. Optimized bond lengths ( $\mathrm{A})$ of DO3A-APB-o and DO3A-ABB- $\alpha$ structures at M062X/6 - $31+\mathrm{G}(\mathrm{d})$ level of theory.

\begin{tabular}{|c|c|c|c|c|c|c|}
\hline Compound & $\begin{array}{c}\text { DO3A-APB-o } \\
\text { (Neutral) }\end{array}$ & $\begin{array}{c}\mathrm{DO} 3 \mathrm{~A}-\mathrm{ABB}-\mathrm{o} \\
\text { (Acidic) }\end{array}$ & $\begin{array}{c}\text { DO3A-APB-o } \\
\text { (Basic) }\end{array}$ & $\begin{array}{c}\mathrm{DO} 3 \mathrm{~A}-\mathrm{UO}_{2} \\
\text { (VI) APB-o } \\
\text { (Neutral) }\end{array}$ & $\begin{array}{c}\mathrm{DO} 3 \mathrm{~A}-\mathrm{UO}_{2} \\
\text { (VI) APB-o } \\
\text { (Acidic) }\end{array}$ & $\begin{array}{c}\mathrm{DO} 3 \mathrm{~A}-\mathrm{UO}_{2} \\
\text { (VI) APB-o } \\
\text { (Basic) }\end{array}$ \\
\hline $\mathrm{d}(\mathrm{N} 1-\mathrm{C} 5)$ & 1.446 & 1.441 & 1.446 & 1.476 & 1.469 & 1.370 \\
\hline $\mathrm{d}(\mathrm{N} 1-\mathrm{C} 10)$ & 1.463 & 1.464 & 1.463 & 1.506 & 1.521 & 1.511 \\
\hline $\mathrm{d}(\mathrm{C} 10-\mathrm{C} 11)$ & 1.534 & 1.532 & 1.535 & 1.564 & 1.571 & 1.577 \\
\hline $\mathrm{d}(\mathrm{N} 2-\mathrm{C} 11)$ & 1.475 & 1.474 & 1.468 & 1.522 & 1.524 & 1.527 \\
\hline d (N2-C12) & 1.455 & 1.457 & 1.456 & 1.477 & 1.479 & 1.482 \\
\hline d (N6-C14) & 1.353 & 1.517 & 1.353 & 1.355 & 1.506 & 1.357 \\
\hline $\mathrm{d}(\mathrm{C} 5-\mathrm{C} 14)$ & 1.527 & 1.517 & 1.528 & 1.535 & 1.505 & 1.533 \\
\hline d (N6-C7) & 1.411 & 1.471 & 1.409 & 1.414 & 1.488 & 1.413 \\
\hline $\mathrm{d}(\mathrm{N} 8-\mathrm{C} 7)$ & - & - & - & - & - & - \\
\hline d (N9-C7) & - & - & - & - & - & - \\
\hline $\mathrm{d}(\mathrm{O}-\mathrm{C} 13)$ & 1.218 & 1.217 & 1.258 & 1.226 & 1.226 & 1.271 \\
\hline $\mathrm{d}(\mathrm{OH}-\mathrm{C} 13)$ & 1.330 & 1.331 & - & 1.363 & 1.363 & - \\
\hline $\mathrm{d}(\mathrm{B}-\mathrm{OH})$ & 1.370 & 1.364 & 1.370 & 1.377 & 1.362 & 1.377 \\
\hline $\mathrm{d}(\mathrm{N} 1-\mathrm{U})$ & - & - & - & 2.544 & 2.570 & 2.543 \\
\hline $\mathrm{d}(\mathrm{N} 2-\mathrm{U})$ & - & - & - & 2.685 & 2.654 & 2.550 \\
\hline d (N3-U) & - & - & - & 2.549 & 2.507 & 2.484 \\
\hline $\mathrm{d}(\mathrm{N} 4-\mathrm{U})$ & - & - & - & 2.544 & 2.507 & 2.492 \\
\hline Compound & $\begin{array}{c}\text { DO3A-ABB- } \alpha \\
\text { (Neutral) }\end{array}$ & $\begin{array}{c}\alpha \mathrm{DO} 3 \mathrm{~A}-\mathrm{ABB}-\alpha \\
\text { (Acidic) }\end{array}$ & $\begin{array}{c}\mathrm{DO} 3 \mathrm{~A}-\mathrm{ABB}-\alpha \\
\text { (Basic) }\end{array}$ & $\begin{array}{c}\mathrm{DO}^{\mathrm{A}-\mathrm{UO}_{2}} \\
\text { (VI) ABB- } \alpha \\
\text { (Neutral) }\end{array}$ & $\begin{array}{c}\mathrm{DO}^{2} \mathrm{~A}-\mathrm{UO}_{2} \\
\text { (VI) } \mathrm{ABB}-\alpha \\
\text { (Acidic) }\end{array}$ & $\begin{array}{c}\mathrm{DO} 3 \mathrm{~A}-\mathrm{UO}_{2} \\
\text { (VI) } \mathrm{ABB}-\alpha \\
\quad \text { (Basic) }\end{array}$ \\
\hline d (N1-C5) & 1.450 & 1.445 & 1.453 & 1.477 & 1.469 & 1.474 \\
\hline $\mathrm{d}(\mathrm{N} 1-\mathrm{C} 10)$ & 1.463 & 1.467 & 1.465 & 1.515 & 1.522 & 1.514 \\
\hline $\mathrm{d}(\mathrm{C} 10-\mathrm{C} 11)$ & 1.534 & 1.530 & 1.533 & 1.569 & 1.568 & 1.576 \\
\hline $\mathrm{d}(\mathrm{N} 2-\mathrm{C} 11)$ & 1.476 & 1.473 & 1.469 & 1.521 & 1.514 & 1.528 \\
\hline d (N2-C12) & 1.456 & 1.457 & 1.456 & 1.469 & 1.464 & 1.483 \\
\hline d (N6-C14) & 1.364 & 1.554 & 1.365 & 1.373 & 1.577 & 1.375 \\
\hline $\mathrm{d}(\mathrm{C} 5-\mathrm{C} 14)$ & 1.524 & 1.507 & 1.525 & 1.532 & 1.505 & 1.531 \\
\hline d (N6-C7) & 1.385 & 1.437 & 1.388 & 1.384 & 1.445 & 1.383 \\
\hline d (N8-C7) & 1.364 & 1.352 & 1.362 & 1.325 & 1.310 & 1.382 \\
\hline d (N9-C7) & 1.310 & 1.298 & 1.310 & 1.382 & 1.367 & 1.325 \\
\hline $\mathrm{d}(\mathrm{O}-\mathrm{C} 13)$ & 1.218 & 1.214 & 1.267 & 1.226 & 1.225 & 1.271 \\
\hline d OH-C13) & 1.330 & 1.347 & - & 1.365 & 1.365 & - \\
\hline $\mathrm{d}(\mathrm{B}-\mathrm{OH})$ & 1.368 & 1.371 & 1.371 & 1.390 & 1.390 & 1.378 \\
\hline $\mathrm{d}(\mathrm{N} 1-\mathrm{U})$ & - & - & - & 2.534 & 2.591 & 2.555 \\
\hline $\mathrm{d}(\mathrm{N} 2-\mathrm{U})$ & - & - & - & 2.677 & 2.658 & 2.555 \\
\hline $\mathrm{d}(\mathrm{N} 3-\mathrm{U})$ & - & - & - & 2.532 & 2.506 & 2.476 \\
\hline $\mathrm{d}(\mathrm{N} 4-\mathrm{U})$ & - & - & - & 2.604 & 2.603 & 2.488 \\
\hline
\end{tabular}


Table 3. Optimized Mulliken charges (Debye) of DO3A-APB-o and DO3A-ABB- $\alpha$ structures.

\begin{tabular}{|c|c|c|c|c|c|c|}
\hline Compound & $\begin{array}{c}\text { DO3A-APB-o } \\
\text { (Neutral) }\end{array}$ & $\begin{array}{l}\text { DO3A-APB-o } \\
\text { (Acidic) }\end{array}$ & $\begin{array}{l}\text { DO3A-APB-o } \\
\text { (Basic) }\end{array}$ & $\begin{array}{c}\mathrm{DO}^{\mathrm{A}}-\mathrm{UO}_{2} \\
\text { (VI) APB-o } \\
\text { (Neutral) }\end{array}$ & $\begin{array}{c}\mathrm{DO} 3 \mathrm{~A}-\mathrm{UO}_{2} \\
\text { (VI) APB-o } \\
\text { (Acidic) }\end{array}$ & $\begin{array}{c}\mathrm{DO} 3 \mathrm{~A}-\mathrm{UO}_{2} \\
\text { (VI) APB-o } \\
\quad \text { (Basic) }\end{array}$ \\
\hline N1 & -0.41 & -0.35 & -0.45 & -0.52 & -0.53 & -0.52 \\
\hline $\mathrm{N} 2$ & -0.62 & -0.60 & -0.40 & -0.50 & -0.51 & -0.50 \\
\hline N3 & -0.31 & -0.24 & -0.39 & -0.53 & -0.53 & -0.50 \\
\hline $\mathrm{N} 4$ & -0.12 & -0.05 & -0.10 & -0.52 & -0.52 & -0.50 \\
\hline C5 & -0.12 & -0.42 & -0.33 & -0.30 & -0.29 & -0.30 \\
\hline N6 & -0.45 & -1.13 & -0.47 & -0.75 & -0.83 & -0.75 \\
\hline C7 & 0.02 & 0.44 & 0.06 & 0.22 & 0.12 & 0.23 \\
\hline N8 & - & - & - & - & - & - \\
\hline N9 & - & - & - & - & - & - \\
\hline $\mathrm{C} 10$ & 0.12 & -0.19 & -0.03 & -0.18 & -0.19 & -0.18 \\
\hline $\mathrm{C} 11$ & -0.61 & -0.24 & -0.54 & -0.17 & -0.17 & -0.19 \\
\hline $\mathrm{C} 12$ & -0.38 & -0.31 & -0.54 & -0.30 & -0.30 & -0.30 \\
\hline $\mathrm{C} 13$ & 0.51 & 0.47 & 0.63 & 0.63 & 0.63 & 0.55 \\
\hline $\mathrm{C} 14$ & 0.34 & 0.56 & 0.55 & 0.62 & 0.61 & 0.62 \\
\hline B & 1.08 & 0.95 & 1.09 & 0.54 & 0.58 & 0.54 \\
\hline U & - & - & - & 1.76 & 1.76 & 1.62 \\
\hline Compound & $\begin{array}{c}\text { DO3A-ABB- } \alpha \\
\text { (Neutral) }\end{array}$ & $\begin{array}{c}\text { DO3A-ABB- } \alpha \\
\text { (Acidic) }\end{array}$ & $\begin{array}{c}\text { DO3A-ABB- } \alpha \\
\text { (Basic) }\end{array}$ & $\begin{array}{c}\mathrm{DO} 3 \mathrm{~A}-\mathrm{UO}_{2} \\
\text { (VI) ABB- } \alpha \\
\text { (Neutral) }\end{array}$ & $\begin{array}{c}\mathrm{DO}^{2} \mathrm{~A}-\mathrm{UO}_{2} \\
\text { (VI) ABB- } \alpha \\
\text { (Acidic) }\end{array}$ & $\begin{array}{c}\mathrm{DO} 3 \mathrm{~A}-\mathrm{UO}_{2} \\
\text { (VI) ABB- } a \\
\quad \text { (Basic) }\end{array}$ \\
\hline N1 & -0.44 & -0.07 & -0.49 & -0.52 & -0.53 & -0.52 \\
\hline $\mathrm{N} 2$ & -0.66 & -0.48 & -0.38 & -0.50 & -0.51 & -0.50 \\
\hline N3 & -0.32 & -0.02 & -0.38 & -0.53 & -0.53 & -0.50 \\
\hline N4 & -0.07 & -0.21 & 0.05 & -0.52 & -0.51 & -0.50 \\
\hline C5 & -0.06 & 0.57 & 0.05 & -0.30 & -0.28 & -0.30 \\
\hline N6 & -0.47 & -0.27 & -0.47 & -0.75 & -0.82 & -0.73 \\
\hline C7 & 1.05 & 0.78 & 0.06 & 0.20 & 0.12 & 0.70 \\
\hline N8 & -0.66 & -0.63 & -0.66 & -0.74 & -0.68 & -0.76 \\
\hline N9 & -0.45 & -0.30 & -0.45 & -0.53 & -0.47 & -0.53 \\
\hline $\mathrm{C} 10$ & -0.25 & 0.41 & -0.27 & -0.20 & -0.20 & -0.18 \\
\hline C11 & -0.19 & 0.12 & -0.20 & -0.20 & -0.18 & -0.19 \\
\hline $\mathrm{C} 12$ & -0.36 & 0.18 & -0.53 & -0.29 & -0.82 & -0.30 \\
\hline $\mathrm{C} 13$ & 0.55 & 0.54 & 0.69 & 0.63 & 0.64 & 0.55 \\
\hline C14 & 0.19 & 0.32 & 0.06 & 0.62 & 0.62 & 0.62 \\
\hline B & 1.04 & 1.07 & 1.10 & 0.50 & 0.50 & 0.50 \\
\hline U & - & - & - & 1.81 & 1.81 & 1.62 \\
\hline
\end{tabular}




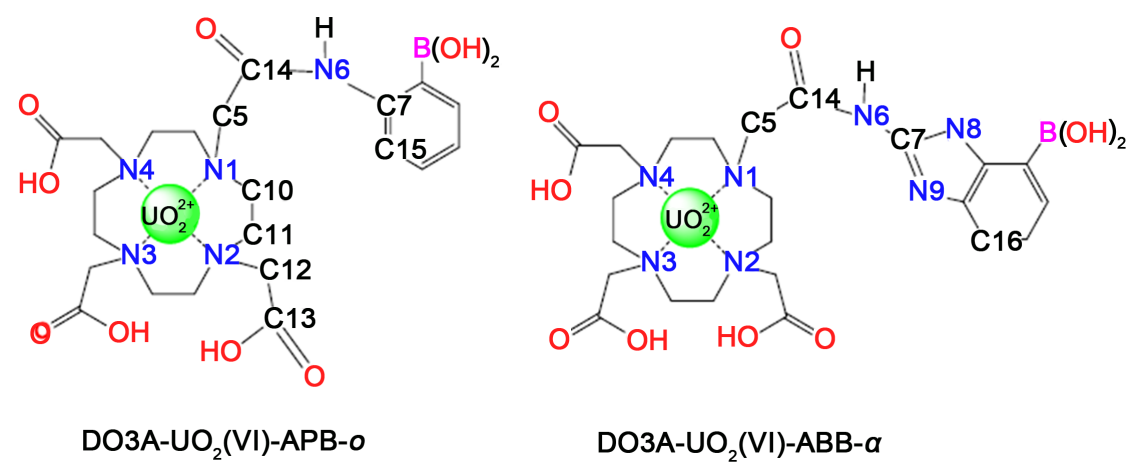

Figure 2. Schematic structures of DO3A-based sensors with/without uranyl ion.

the charges of nitrogen atoms in theDO3A ring reduce in the acidic medium and are in the range of -0.60 to -0.05 , when the nitrogen charges in the other medium are compared. For all three medium, these charges are close to reaching -0.50 after the bonded with the uranyl ion. The charge of amide nitrogen is about -0.40 in the neutral and basic medium while it increases to -1.13 in the acidic medium. In the DO3A-UO $\mathrm{UO}_{2}(\mathrm{VI})-\mathrm{APB}-\mathrm{o}$ complexes, it increases to -0.75 in the neutral and basic medium while it decreases to -0.85 in the acidic medium. The charge of $\mathrm{C} 5$ carbon atom linking DO3A to amide falls from -0.40 in the acidic medium to -0.29 in the $\mathrm{UO}_{2}$ complexes. The charge of the boron atom in the DO3A-APB-o structures decreases from -1.00 to -0.55 in $\mathrm{DO}^{\mathrm{A}-\mathrm{UO}_{2}}$ (VI)-APB-o structures. The uranium (VI) ion charge is in the range of 1.75 to 1.61. Similar trends and values have been found forDO3A-ABB- $\alpha$ and $\mathrm{DO} 3 \mathrm{~A}-\mathrm{UO}_{2}$ (VI)-ABB- $\alpha$ structures in all three mediums.

The highest occupied molecular orbital (HOMO) and lowest unoccupied molecular orbital (LUMO) are very important parameters as a measure of relative stability and reactivity. The energies of frontier orbitals, total energies $\left(\mathrm{E}_{\mathrm{T}}\right)$, chemical hardness $(\eta)$, electronic chemical potentials $(\mu)$, chemical softness (S) and electrophilicity $(\omega)$ values are listed in Table 4.

When the total energy values of the DO3A-APB-o are examined,it can be seen that the energy stability decreases in these order DO3A-APB-o acidic $>$ DO3A-APB-o neutral > DO3A-APB-o basic media. The same trend has been for the DO3A-ABB- $\alpha$, $\mathrm{DO}_{3 \mathrm{~A}-\mathrm{UO}}$ (VI)-ABB- $\alpha$ and DO3A-UO $(\mathrm{VI})-\mathrm{APB}-\mathrm{o}$ series structures. The most stable structures are in acidic media.

When the total energy values of the DO3A-APB-o are examined, it can be seen that the energy stability decreases in these order DO3A-APB-o acidic > DO3A-APB-o neutral > DO3A-APB-o basic media. The same trend has been seen for the DO3A-ABB- $\alpha$, DO3A-UO ${ }_{2}$ (VI)-ABB- $\alpha$ and DO3A-UO $(\mathrm{VI})-\mathrm{APB}-\mathrm{o}$ series structures. The most stable structures have been found in the acidic media.

A large HOMO-LUMO energy gap has been associated with high stability of structures. For DO3A-APB-o and DO3A-ABB- $\alpha$ series, the HOMO-LUMO energy gap of all calculated structures decrease to about $2 \mathrm{eV}$ by binding uranyl ion and their lower one is counter to neutral medium. On the other hands, their uranyl structures have a largest band gap in acidic medium. Among the calcu- 
Table 4. Values of the global reactivity descriptors of the systems (eV).

\begin{tabular}{|c|c|c|c|c|c|c|c|c|c|c|c|}
\hline Compound & $\mathrm{E}_{\mathrm{T}}$ & $\mathrm{E}_{\text {Номо }}$ & $\mathrm{E}_{\text {LUMO }}$ & $\mathrm{E}_{\mathrm{GAP}}$ & I & A & $x$ & $\eta$ & S & $\mu$ & $\omega$ \\
\hline DO3A-APB-o (N) & $-1,150,446.42$ & -7.31 & -0.19 & 7.12 & 7.31 & 0.19 & 3.75 & 3.56 & 0.28 & -3.75 & 1.97 \\
\hline DO3A-APB-o (A) & $-1,150,690.41$ & -7.47 & -0.83 & 6.64 & 7.47 & 0.83 & 4.15 & 3.32 & 0.30 & -4.15 & 2.60 \\
\hline DO3A-APB-o (B) & $-1,149,613.33$ & -6.97 & -1.24 & 5.73 & 6.97 & 1.24 & 4.10 & 2.86 & 0.35 & -4.10 & 2.94 \\
\hline DO3A-UO $2(\mathrm{VI})-\mathrm{APB}-\mathrm{o}(\mathrm{N})$ & $-17,342,528.18$ & -7.51 & -3.11 & 4.40 & 7.51 & 3.11 & 5.31 & 2.20 & 0.45 & -5.31 & 6.41 \\
\hline DO3A-UO $2(\mathrm{VI})-\mathrm{APB}-\mathrm{o}(\mathrm{A})$ & $-17,342,776.59$ & -8.17 & -3.22 & 4.95 & 8.17 & 3.22 & 5.70 & 2.48 & 0.40 & -5.70 & 6.55 \\
\hline DO3A-UO $_{2}(\mathrm{VI})-\mathrm{APB}-\mathrm{o}(\mathrm{B})$ & $-17,341,682.81$ & -7.34 & -2.64 & 4.70 & 7.34 & 2.64 & 4.99 & 2.35 & 0.43 & -4.99 & 5.30 \\
\hline DO3A-ABB- $\alpha(\mathrm{N})$ & $-1,243,051.98$ & -7.44 & -0.36 & 7.08 & 7.44 & 0.36 & 3.90 & 3.54 & 0.28 & -3.90 & 2.15 \\
\hline DO3A-ABB- $\alpha(\mathrm{A})$ & $-1,243,294.62$ & -7.52 & -0.69 & 6.83 & 7.52 & 0.69 & 4.10 & 3.41 & 0.29 & -4.10 & 2.47 \\
\hline $\mathrm{DO} 3 \mathrm{~A}-\mathrm{ABB}-\alpha(\mathrm{B})$ & $-1,242,223.34$ & -7.08 & -0.25 & 6.83 & 7.08 & 0.25 & 3.67 & 3.42 & 0.29 & -3.67 & 1.97 \\
\hline DO3A-UO ${ }_{2}(\mathrm{VI})-\mathrm{ABB}-\alpha(\mathrm{N})$ & $-17,435,128.24$ & -7.26 & -3.19 & 4.07 & 7.26 & 3.19 & 5.23 & 2.04 & 0.49 & -5.23 & 6.71 \\
\hline $\mathrm{DO} 3 \mathrm{~A}-\mathrm{UO}_{2}(\mathrm{VI})-\mathrm{ABB}-\alpha(\mathrm{A})$ & $-17,435,369.38$ & -7.95 & -3.31 & 4.64 & 7.95 & 3.31 & 5.63 & 2.32 & 0.43 & -5.63 & 6.83 \\
\hline $\mathrm{DO} 3 \mathrm{~A}-\mathrm{UO}_{2}(\mathrm{VI})-\mathrm{ABB}-\alpha(\mathrm{B})$ & $-17,434,284.04$ & -7.21 & -2.66 & 4.55 & 7.21 & 2.66 & 4.93 & 2.27 & 0.44 & -4.93 & 5.35 \\
\hline
\end{tabular}

lated chemical hardness $(\eta)$, DO3A-APB-o in basic media, DO3A-ABB- $\alpha$ in acidic media and both DO3A-UO ${ }_{2}$ (VI)-APB-o and DO3A-UO ${ }_{2}$ (VI)-ABB- $\alpha$ structures in neutral media have lower values than the rest of the their series and these result indicating that they are kinetically more stable and less reactive than the other their structures.

\subsection{Absorption Spectra Analysis}

The absorption spectrum of structures has been calculated using Time-Dependent DFT calculations same levels of theory in water phase three indicated media. The calculated absorption bands are summarized in Figure 3.

For DO3A-APB-o compound in the indicated mediums absorption bands outside the visible region and are range of $205 \mathrm{~nm}-230 \mathrm{~nm}$. It can be seen from the Figure 3, when DO3A-APB-o bonded with uranyl ion, $\mathrm{DO}^{\mathrm{A}}-\mathrm{UO}_{2}$ (VI)-APB-o, oscillator strength decreases and shift to long wavelength. At the beginning, a single peak has seen and two flattened peaks have formed at with the addition of uranyl ion. These peaks are in the range of $384-392 \mathrm{~nm}$ and $645-695 \mathrm{~nm}$. For DO3A-ABB- $\alpha$, calculated absorption bands are range in $200-264 \mathrm{~nm}$ within three indicated media. $\mathrm{DO} 3 \mathrm{~A}-\mathrm{UO}_{2}(\mathrm{VI})-\mathrm{ABB}-\alpha$ gives the same trend and these peaks are in the range of $374-393 \mathrm{~nm}$ and $645-688 \mathrm{~nm}$. Consequently, when DO3A-APB-o structure bonds with uranyl ion, the maximum shift is in the neutral medium, whereas maximum shifts of DO3A-ABB- $\alpha$ with uranyl ion are in the basic medium.

\subsection{Infrared and NMR Spectra Analysis}

In this section, the changes in ${ }^{13} \mathrm{C}-\mathrm{NMR}$ and ${ }^{1} \mathrm{H}-\mathrm{NMR}$ chemical shift values of the all the calculated complexes formed by the binding of uranyl ions have been summarized in Table 5 and Table 6. 
Table 5. ${ }^{13} \mathrm{C}-\mathrm{NMR}$ chemical shifts (ppm) of DO3A-APB-o and DO3A-ABB- $\alpha$ and their structures with uranyl ion.

\begin{tabular}{|c|c|c|c|c|c|c|}
\hline Compound & $\begin{array}{l}\text { DO3A-APB-o } \\
\text { (Neutral) }\end{array}$ & $\begin{array}{c}\text { DO3A-APB-o } \\
\text { (Acidic) }\end{array}$ & $\begin{array}{c}\text { DO3A-APB-o } \\
\text { (Basic) }\end{array}$ & $\begin{array}{c}\mathrm{DO}^{\mathrm{A}-\mathrm{UO}_{2}} \\
\text { (VI) APB-o } \\
\text { (Neutral) }\end{array}$ & $\begin{array}{c}\mathrm{DO} 3 \mathrm{~A}-\mathrm{UO}_{2} \\
\text { (VI) APB-o } \\
\text { (Acidic) }\end{array}$ & $\begin{array}{c}\mathrm{DO}_{\mathrm{A}-\mathrm{UO}_{2}} \\
\text { (VI) APB-o } \\
\text { (Basic) }\end{array}$ \\
\hline C5 & 62.9 & 63.8 & 63.6 & 59.8 & 64.6 & 60.7 \\
\hline $\mathrm{C} 7$ & 158.6 & 145.2 & 158.3 & 158.7 & 152.3 & 159.7 \\
\hline $\mathrm{C} 10$ & 57.4 & 58.0 & 56.6 & 59.1 & 56.2 & 53.8 \\
\hline $\mathrm{C} 11$ & 46.7 & 48.7 & 45.9 & 64.6 & 65.1 & 59.6 \\
\hline $\mathrm{C} 12$ & 61.8 & 62.9 & 66.2 & 71.4 & 70.5 & 70.2 \\
\hline $\mathrm{C} 13$ & 191.3 & 189.7 & 182.6 & 189.3 & 189.2 & 191.4 \\
\hline $\mathrm{C} 14$ & 18.3 & 196.5 & 187.9 & 185.3 & 195.7 & 185.7 \\
\hline Compound & $\begin{array}{l}\text { DO3A-ABB- } \alpha \\
\quad \text { (Neutral) }\end{array}$ & $\begin{array}{c}\text { DO3A-ABB- } \alpha \\
\text { (Acidic) }\end{array}$ & $\begin{array}{l}\text { DO3A-ABB- } \alpha \\
\text { (Basic) }\end{array}$ & $\begin{array}{c}\mathrm{DO}^{2} \mathrm{~A}-\mathrm{UO}_{2} \\
\text { (VI) ABB- } \alpha \\
\text { (Neutral) }\end{array}$ & $\begin{array}{c}\mathrm{DO}^{2} \mathrm{~A}-\mathrm{UO}_{2} \\
\text { (VI) ABB- } \alpha \\
\text { (Acidic) }\end{array}$ & $\begin{array}{c}\mathrm{DO}_{\mathrm{AA}-\mathrm{UO}_{2}} \\
\text { (VI) ABB- } \alpha \\
\text { (Basic) }\end{array}$ \\
\hline C5 & 64.5 & 63.9 & 64.3 & 61.8 & 64.5 & 61.0 \\
\hline C7 & 149.2 & 142.4 & 154.5 & 61.8 & 144.6 & 155.3 \\
\hline $\mathrm{C} 10$ & 52.0 & 53.7 & 49.0 & 54.1 & 54.4 & 54.6 \\
\hline $\mathrm{C} 11$ & 63.7 & 62.6 & 61.6 & 54.7 & 56.8 & 60.6 \\
\hline $\mathrm{C} 12$ & 61.1 & 62.3 & 65.9 & 61.9 & 62.8 & 70.8 \\
\hline $\mathrm{C} 13$ & 190.1 & 189.9 & 192.1 & 188.0 & 187.7 & 191.2 \\
\hline $\mathrm{C} 14$ & 185.0 & 184.0 & 186.6 & 188.0 & 184.3 & 184.0 \\
\hline
\end{tabular}

Table 6. ${ }^{1} \mathrm{H}-\mathrm{NMR}$ chemical shifts (ppm) of DO3A-APB-o and DO3A-ABB- $\alpha$ and their structures with uranyl ion.

\begin{tabular}{ccccccc}
\hline Compound & $\begin{array}{c}\text { DO3A-APB-o } \\
\text { (Neutral) }\end{array}$ & $\begin{array}{c}\text { DO3A-APB-o } \\
\text { (Acidic) }\end{array}$ & $\begin{array}{c}\text { DO3A-APB-o } \\
\text { (Basic) }\end{array}$ & $\begin{array}{c}\text { DO3A-UO } \\
\text { (VI) APB-o } \\
\text { (Neutral) }\end{array}$ & $\begin{array}{c}\text { DO3A-UO } \\
\text { (VI) APB-o } \\
\text { (Acidic) }\end{array}$ & $\begin{array}{c}\text { DO3A-UO } \\
\text { (VI) APB-o } \\
\text { (Basic) }\end{array}$ \\
\hline BO-H & 4.9 & 5.4 & 4.8 & 6.0 & 6.7 & 5.7 \\
N6-H & 11.0 & 6.4 & 11.2 & 12.4 & 8.1 & 11.8 \\
N8-H & - & - & - & - & - & - \\
C14-H & 7.0 & 7.5 & - & 8.3 & 8.3 & - \\
C10-H & 2.3 & 2.6 & 2.2 & 3.5 & 3.3 & 3.8 \\
C11-H & 3.1 & 2.9 & 3.3 & 3.9 & 4.1 & 3.9 \\
C15-H & 9.3 & 7.8 & 9.2 & 8.3 & 8.4 & 9.5 \\
\hline
\end{tabular}

\begin{tabular}{|c|c|c|c|c|c|c|}
\hline Compound & $\begin{array}{c}\text { DO3A-ABB- } \alpha \\
\text { (Neutral) }\end{array}$ & $\begin{array}{l}\text { DO3A-ABB- } \alpha \\
\text { (Acidic) }\end{array}$ & $\begin{array}{c}\text { DO3A-ABB- } \alpha \\
\text { (Basic) }\end{array}$ & $\begin{array}{c}\mathrm{DO}^{\mathrm{A}}-\mathrm{UO}_{2} \\
\text { (VI) ABB- } \alpha \\
\text { (Neutral) }\end{array}$ & $\begin{array}{c}\mathrm{DO} 3 \mathrm{~A}-\mathrm{UO}_{2} \\
\text { (VI) ABB- } \alpha \\
\text { (Acidic) }\end{array}$ & $\begin{array}{c}\mathrm{DO} 3 \mathrm{~A}-\mathrm{UO}_{2} \\
\text { (VI) ABB- } \alpha \\
\quad \text { (Basic) }\end{array}$ \\
\hline BO-H & 5.1 & 6.1 & 5.1 & 5.9 & 5.9 & 5.8 \\
\hline N6-H & 9.4 & 11.1 & 9.5 & 8.4 & 8.3 & 8.0 \\
\hline N8-H & 9.9 & 10.6 & 10.2 & 10.0 & 11.1 & 9.8 \\
\hline C14-H & 9.4 & 11.5 & - & 8.3 & 8.3 & - \\
\hline $\mathrm{C} 10-\mathrm{H}$ & 3.6 & 2.4 & 2.2 & 3.7 & 3.3 & 3.8 \\
\hline $\mathrm{C} 11-\mathrm{H}$ & 3.0 & 2.5 & 3.3 & 3.3 & 3.9 & 2.7 \\
\hline $\mathrm{C} 15-\mathrm{H}$ & 8.5 & 9.0 & 8.6 & 8.1 & 8.4 & 8.0 \\
\hline
\end{tabular}





Figure 3. Computed UV-Vis absorption bands. The $\lambda_{\max }$ values of structures also have been indicated on the graphs.

Firstly, the chemical shift of TMS (tetramethylsilane) has been calculated with the same level of theory in order to carry out the NMR calculations. Subsequently, the NMR spectrum of the structures has been calculated and the difference between the two values gives the final result. In the calculated ${ }^{13} \mathrm{C}-\mathrm{NMR}$ spectra, a chemical shift in the C5 atom ca. $0.5-3 \mathrm{ppm}$ is observed in the high field while C11 carbon (ca. $14-18$ ppm) has low field for DO3A-APB-o structures. The same trend exists for C12 (ca. 10 ppm). Similarly, C5 and C11 carbon atoms belong to DO3A-ABB- $\alpha$ structures have been found in the high field ca. $1 \mathrm{ppm}$ and ca. 5 - 9 ppm, respectively. There is no significant change in other carbon atoms, but there is a low shift in C10 atom, ca. 2 - 5 ppm. In the calculated ${ }^{1} \mathrm{H}-\mathrm{NMR}$ spectra, the corresponding proton peaks connected to C10, C11 (ca. $1 \mathrm{ppm}$ ) and N6 (ca. $2 \mathrm{ppm}$ ) of DO3A-APB-o structures have shifted to the low field. In the DO3A-ABB- $\alpha$ structures, there are only high field shift in N6-H and $\mathrm{C} 10-\mathrm{H}$ peaks, ca. 1 - 2 ppm, there is no big differences for the other peaks.

The calculated harmonic vibrations and most characteristic peaks of the compounds are also included in this part and given in Table 7. The main effect on DO3A-UO $\mathrm{U}_{2}$ (VI)-APB-o on calculated IR-spectrum is seen at the $\mathrm{BO}-\mathrm{H}$ and N6-H stretching vibrations for all mediums. The bond stretching vibrations of BO-H and N6-H occur in the region of $3565 \mathrm{~cm}^{-1}$ and $3234 \mathrm{~cm}^{-1}$ in the neutral 
Table 7. Selected and calculated vibrational frequency $\left(\mathrm{cm}^{-1}\right)$.

\begin{tabular}{|c|c|c|c|c|c|c|}
\hline Compound & $\begin{array}{c}\text { DO3A-APB-o } \\
\text { (Neutral) }\end{array}$ & $\begin{array}{c}\text { DO3A-APB-o } \\
\text { (Acidic) }\end{array}$ & $\begin{array}{c}\text { DO3A-APB-o } \\
\text { (Basic) }\end{array}$ & $\begin{array}{c}\text { DO3A-UO } \\
\text { (VI) APB-o } \\
\text { (Neutral) }\end{array}$ & $\begin{array}{c}\mathrm{DO} 3 \mathrm{~A}-\mathrm{UO}_{2} \\
\text { (VI) APB-o } \\
\text { (Acidic) }\end{array}$ & $\begin{array}{c}\mathrm{DO}^{\mathrm{A}}-\mathrm{UO}_{2} \\
\text { (VI) APB-o } \\
\text { (Basic) }\end{array}$ \\
\hline BO-H & 3787 & -3777 & 3789 & 3565 & 3545 & 3535 \\
\hline $\mathrm{C} 13-\mathrm{OH}$ & 3436 & 3475 & - & 3448 & 3456 & - \\
\hline $\begin{array}{c}\text { Aromatic } \\
\text { C-H }\end{array}$ & 3245 & 3247 & 3241 & 3309 & 3274 & 3263 \\
\hline $\mathrm{N} 6-\mathrm{H}$ & 3473 & 3396 & 3469 & 3234 & 3282 & 3248 \\
\hline $\mathrm{C} 10-\mathrm{H}$ & 3129 & 3175 & 3163 & 3200 & 3202 & 3100 \\
\hline $\mathrm{C} 13-\mathrm{O}$ & 1795 & 1800 & 1583 & 1755 & 1750 & 1591 \\
\hline C14-O & 1711 & 1920 & 1709 & 1682 & 1800 & 1684 \\
\hline $\begin{array}{c}\text { Aromatic } \\
\text { C-C }\end{array}$ & 1687 & 1660 & 1687 & 1641 & 1701 & 1643 \\
\hline C14-N6 & 1584 & 1550 & 1521 & 1607 & 1659 & 1606 \\
\hline $\mathrm{U}-\mathrm{O}$ & - & - & - & 864 & 861 & 825 \\
\hline U-N & - & - & - & 742 & 740 & 756 \\
\hline B-C & 1379 & 1381 & 1367 & 1372 & 1383 & 1367 \\
\hline Compound & $\begin{array}{c}\text { DO3A-ABB- } \alpha \\
\text { (Neutral) }\end{array}$ & $\begin{array}{c}\text { DO3A-ABB- } \alpha \\
\text { (Acidic) }\end{array}$ & $\begin{array}{c}\text { DO3A-ABB- } \alpha \\
\text { (Basic) }\end{array}$ & $\begin{array}{c}\mathrm{DO} 3 \mathrm{~A}-\mathrm{UO}_{2} \\
\text { (VI) ABB- } \alpha \\
\text { (Neutral) }\end{array}$ & $\begin{array}{c}\mathrm{DO}^{\mathrm{A}}-\mathrm{UO}_{2} \\
\text { (VI) ABB- } \alpha \\
\quad \text { (Acidic) }\end{array}$ & $\begin{array}{l}\mathrm{DO} 3 \mathrm{~A}-\mathrm{UO}_{2} \\
\text { (VI) } \mathrm{ABB}-\alpha \\
\quad \text { (Basic) }\end{array}$ \\
\hline BO-H & 3799 & 3712 & 3782 & 3541 & 3533 & 3539 \\
\hline $\mathrm{C} 13-\mathrm{OH}$ & 3476 & 3450 & - & 3441 & 3452 & - \\
\hline $\begin{array}{c}\text { Aromatic } \\
\text { C-H }\end{array}$ & 3236 & 3235 & 3232 & 3267 & 3265 & 3252 \\
\hline N6-H & 3491 & 3366 & 3489 & 3495 & 3282 & 3501 \\
\hline $\mathrm{C} 10-\mathrm{H}$ & 3160 & 3167 & 3194 & 3188 & 3184 & 3151 \\
\hline $\mathrm{C} 13-\mathrm{O}$ & 1786 & 1799 & 1587 & 1742 & 1746 & 1587 \\
\hline $\mathrm{C} 14-\mathrm{O}$ & 1755 & 1959 & 1756 & 1712 & 1871 & 1751 \\
\hline $\begin{array}{c}\text { Aromatic } \\
\text { C-C }\end{array}$ & 1686 & 1697 & 1690 & 1689 & 1679 & 1688 \\
\hline C14-N6 & 1589 & 1591 & 1584 & 1590 & 1506 & 1572 \\
\hline $\mathrm{U}-\mathrm{O}$ & - & - & - & 867 & 880 & 824 \\
\hline U-N & - & - & - & 724 & 740 & 767 \\
\hline B-C & 1405 & 1412 & 1382 & 1382 & 1379 & 1385 \\
\hline N8-H & 3613 & 3583 & 3533 & 3533 & 3476 & 3530 \\
\hline
\end{tabular}

medium. The differences in between DO3A-APB-o and DO3A-UO $(\mathrm{VI})-\mathrm{APB}-\mathrm{o}$ of BO-H stretching modes are among 222 and $254 \mathrm{~cm}^{-1}$ and the $\mathrm{N} 6-\mathrm{H}$ one is in between $114-239 \mathrm{~cm}^{-1}$ in neutral and acidic medium, appears in a lower frequency. In $\mathrm{DO} 3 \mathrm{~A}-\mathrm{UO}_{2}(\mathrm{VI})-\mathrm{ABB}-\alpha$ structures, the bond stretching vibrations of $\mathrm{BO}-\mathrm{H}$ and $\mathrm{N} 6-\mathrm{H}$ occur in the region of $3541 \mathrm{~cm}^{-1}$ and $3495 \mathrm{~cm}^{-1}$ in the neutral 
medium. When the stretching mode of $\mathrm{BO}-\mathrm{H}$ passing from DO3A-APB-o to DO3A-UO ${ }_{2}(\mathrm{VI})-\mathrm{ABB}-\alpha$ structures have been shifted to lower frequency (180 - 258 $\left.\mathrm{cm}^{-1}\right)$. There is no significant difference in the basic medium at N8-H stretching vibration frequency, whereas a shift has been found at about $80-100 \mathrm{~cm}^{-1}$ in the neutral and acidic medium.

\section{Conclusion}

Our theoretical work represents structural and spectral properties of newly designed DO3A-based structures sensitive to $\mathrm{UO}_{2}^{2+}$. The structures have been optimized in the gas phase and water phase at three levels of theory (M062X, B3LYP and B3PW9/6 - $31 \mathrm{~g}+(\mathrm{d})$ ) for comparison with experimental value, after that M062X/6 - $31 \mathrm{~g}+(\mathrm{d})$ method has been chosen for all remaining calculations. The calculations have been shown that ortho position of boronic acid is the most stable within all calculated structures. By examining the diameter dimension of the structural features, it has been seen that the linking of the uranium causes the increase of the total diameter up to about $1 \AA$. The relative stability of the studied structures showed that all series are more stable in the acidic medium. The calculated HOMO-LUMO gap results manifest that linking of $\mathrm{UO}_{2}^{2+}$ ion to designed structures has caused the decrease. The theoretical electronic spectrum of DO3A-UO $\mathrm{UO}_{2}$ (VI)-APB-o gives bands at 392 and $687 \mathrm{~nm}$ in neutral medium. DO3A-UO 2 (VI)-ABB- $\alpha$ structure has two main bands at 393 and $650 \mathrm{~nm}$. Both structures show red shift and are located within the visible region by binding $\mathrm{UO}_{2}^{2+}$ ion. The notable shifts in NMR spectrums have been found on $\alpha$-carbon of carbonyl group, ring carbons and amide protons. In IR spectrum, the prominent peaks belong to $\mathrm{BO}-\mathrm{H}$ and $\mathrm{N} 6-\mathrm{H}$ stretching vibrations of calculated structures.

\section{Acknowledgements}

This work was supported by The Scientific and Technological Research Council of Turkey (TÜBİTAK) (No: 115Z504). The calculations were performed on Turkish Academic Network and Information Center (ULAKBİM).

\section{References}

[1] Ishihara, K., and Yamamoto, H. (1999) Arylboron Compounds as Acid Catalysts in Organic Synthetic Transformations. European Journal of Organic Chemistry, 1999, 527-538, https://doi.org/10.1002/(SICI)1099-0690(199903)1999:3<527::AID-EJOC527>3.0.C $\underline{\mathrm{O} ; 2-\mathrm{R}}$

[2] Petasis, N.A. and Zavialov, I.A. (1997) A New and Practical Synthesis of A-Amino Acids From Alkenyl Boronic Acids. Journal of the American Chemical Society, 119, 445-446. https://doi.org/10.1021/ja963178n

[3] Miyaura, N. and Suzuki, A. (1997) Palladium-Catalyzed Cross-Coupling Reactions of Organoboron Compounds. Chemical Reviews, 95, 2457-2483.

https://doi.org/10.1021/cr00039a007 
[4] Latta, R., Springsteen, G. and Wang, B. (2001) Development and Synthesis of an Arylboronic Acid-Based Solid-Phase Amidation Catalyst. Synthesis, 2001, 1611-1613. https://doi.org/10.1055/s-2001-16758

[5] James, T.D., Sandanayake, S. and Shinkai, S. (1996) Saccharide Sensing with Molecular Receptors Based on Boronic Acid. Angewandte Chemie International Edition, 35, 1910-1922. https://doi.org/10.1002/anie.199619101

[6] Liua, S., Shia, F., Zhao, B., Chena, L. and Sua, X. (2013) 3-Aminophenyl Boronic Acid-Functionalized CuInS 2 Quantum Dots as A Near Infrared Fluorescence Probe for the Determination of Dopamine. Biosensors \& Bioelectronics, 47, 379-384. https://doi.org/10.1016/j.bios.2013.03.055

[7] DiCesare, N. and Lakowicz, J.R. (2002) New Sensitive and Selective Fluorescent Probes for Fluoride Using Boronic Acids. Analytical Biochemistry, 301, 111-116. https://doi.org/10.1006/abio.2001.5476

[8] DiCesare, N. and Lakowicz, J.R. (2001) Spectral Properties of Fluorophores Combining the Boronic Acid Group with Electron Donor or Withdrawing Groups. Implication in the Development of Fluorescence Probes for Saccharides. Journal of Physical Chemistry A, 105, 6834-6840. https://doi.org/10.1021/jp010076x

[9] Barge, A., Cappelletti, E., Cravotto, G., Ferrigato, A., Lattuada, L., Marinonib, F. and Tei, L. (2009) Synthesis of Functionalised HP-DO3A Chelating Agents for Conjugation to Biomolecules. Organic \& Biomolecular Chemistry, 7, 3810-3816. https://doi.org/10.1039/b905369g

[10] Riesen, A., Zehnder, M. and Kaden, T.A. (1986) Metal Complexes of Macrocyclic Ligands. Part XXIII. Synthesis, Properties, and Structures of Mononuclear Complexes with 12- and 14-Membered Tetraazamacrocycle- $N, N^{\prime}, N^{\prime \prime}, N^{\prime \prime}$-Tetraacetic Acids. Helvetica Chimica Acta, 69, 2067-2073. https://doi.org/10.1002/hlca.19860690830

[11] Rösch, F., Herzog, H., Stolz, B., Brockmann, J., Köhle, M., Mühlensiepen, H., Marbach, P. and Gärtner, H.W. (1999) Uptake Kinetics of the Somatostatin Receptor Ligand [86Y]DOTA-Dphe1-Tyr3-Octreotide ([86Y]SMT487) using Positron Emission Tomography in Non-Human Primates and Calculation of Radiation Doses of The 90Y-Labelled Analogue. European Journal of Nuclear Medicine, 26, 358-366. https://doi.org/10.1007/s002590050398

[12] Kumar, K., Chang, C.A., Francesconi, L.C., Dischino, D.D., Malley, M.F., Gougoutas, J.Z. and Tweedle, M.F. (1994) Synthesis, Stability, and Structure of Gadolinium(III) and Yttrium(III) Macrocyclic Poly(Amino Carboxylates). Inorganic Chemistry, 33, 3567-3575. https://doi.org/10.1021/ic00094a021

[13] Loncin, M.F., Desreux, J.F. and Merciny, E. (1986) Coordination of Lanthanides by Two Polyamino Poly Carboxylic Macrocycles: Formation of Highly Stable Lanthanide Complexes. Inorganic Chemistry, 25, 2646-2648.

[14] Iglesias, C.P. (2012) The Solution Structurea nd Dynamics of MR Probes Based on Lanthanide(III) Dota as Investigated by DFT and NMR Spectroscopy. European Journal of Inorganic Chemistry, 12, 2023-2033. https://doi.org/10.1002/ejic.201101164

[15] Fernandez, M., Bastida, R., Macias, A., Perez-Lourido, P., Iglesias, C.P. and Valencia, L. (2006) Lanthanide(III) Complexes with a Tetrapyridine Pendant-Armed Macrocyclic Ligand: ${ }^{1} \mathrm{H}$ NMR Structural Determination in Solution, X-Ray Diffraction, and Density-Functional Theory Calculations. Inorganic Chemistry, 45, 4484-4496. https://doi.org/10.1021/ic0603508

[16] Frisch, M.J., Trucks, G.W., Schlegel, H.B., Scuseria, G.E., Robb, M.A., Cheeseman, 
J.R., Scalmani, G., Barone, V., Mennucci, B., Petersson, G.A., Nakatsuji, H., Caricato, M., Li, X., Hratchian, H.P., Izmaylov, A.F., Bloino, J., Zheng, G., Sonnenberg, J.L., Hada, M., Ehara, M., Toyota, K., Fukuda, R., Hasegawa, J., Ishida, M., Nakajima, T., Honda, Y., Kitao, O., Nakai, H., Vreven, T., Montgomery, J.A., Peralta, J.E., Ogliaro, F., Bearpark, M., Heyd, J.J., Brothers, E., Kudin, K.N., Staroverov, V.N., Kobayashi, R., Normand, J., Raghavachari, K., Rendell, A., Burant, J.C., Iyengar, S.S., Tomasi, J., Cossi, M., Rega, N., Millam, J.M., Klene, M., Knox, J.E., Cross, J.B., Bakken, V., Adamo, C., Jaramillo, J., Gomperts, R., Stratmann, R.E., Yazyev, O., Austin, A.J., Cammi, R., Pomelli, C., Ochterski, J.W., Martin, R.L., Morokuma, K., Zakrzewski, V.G., Voth, G.A., Salvador, P., Dannenberg, J.J., Dapprich, S., Daniels, A.D., Farkas, Ö., Foresman, J.B., Ortiz, J.V., Cioslowski, J. and Fox, D.J. (2009) Gaussian 09, Revis. D.01.

[17] Dennington, R.D., Keith, T.A. and Millam, J.M. (2008) GaussView 5.0.8, Gaussian.

[18] https://www.chemcraftprog.com

[19] Walker, M., Harvey, A., Sen, A. and Dessent, C. (2013) Performance of M06, M06-2X, and M06-HF Density Functionals for Conformationally Flexible Anionic Clusters: M06 Functionals Perform Better than B3LYP for a Model System with Dispersion and Ionic Hydrogen-Bonding Interactions. The Journal of Physical Chemistry A, 117, 12590-12600. https://doi.org/10.1021/jp408166m

[20] Rives, T.J. and Jorgensen, W.L. (2008) Performance of B3LYP Density Functional Methods for a Large Set of Organic Molecules. Journal of Chemical Theory and Computation, 4, 297-306. https://doi.org/10.1021/ct700248k

[21] Govindarajana, M., Ganasanb, K., Periandyc, S. and Mohand, S. (2010) DFT (LSDA, B3LYP and B3PW91) Comparative Vibrational Spectroscopic Analysis of Acetonaphthone. Spectrochimica Acta Part A, 76, 12-21.

[22] Natrajan, L.S. (2012) The First Structural and Spectroscopic Study of a Paramagnetic 5f DO3A Complex. Dalton Tran, 41, 13167. https://doi.org/10.1039/c2dt30573a

[23] Del Bene, J.E. (1995) Properties of Hydrogen-Bonded Complexes Obtained from the B3LYP Functional with $6-31 G(d, p)$ and $6-31+G(d, p)$ Basis Sets: Comparison with MP2/6-31+G(d,p) Results and Experimental Data. The Journal of Physical Chemistry, 99, 10705-1070. https://doi.org/10.1021/j100027a005

[24] Pantazis, D.A., Chen, X., Landi,s C.R. and Neese, F. (2008) All-Electron Scalar Relativistic Basis Sets for Third-Row Transition Metal Atoms. Journal of Chemical Theory and Computation, 4, 908-919. https://doi.org/10.1021/ct800047t

[25] Marenich, A.V., Cramer, C.J. and Truhlar, D.G. (2009) Universal Solvation Model Based on Solute Electron Density and on Acontinuum Model of the Solvent Defined by the Bulk Dielectric Constant and Atomic Surface Tensions. The Journal of Physical Chemistry B, 113, 6378-6396. https://doi.org/10.1021/jp810292n 